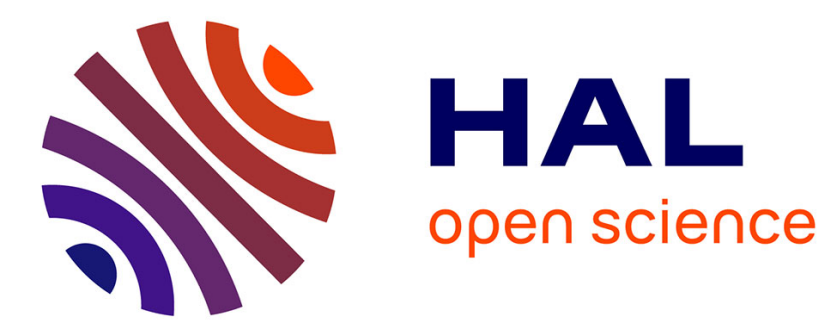

\title{
Steady state behavior of a multipolar plasma device
}

M Carrère, Laurence Chérigier-Kovacic, C Arnas-capeau, G Bachet, F Doveil

\section{To cite this version:}

M Carrère, Laurence Chérigier-Kovacic, C Arnas-capeau, G Bachet, F Doveil. Steady state behavior of a multipolar plasma device. Review of Scientific Instruments, 1996, 67, pp.4124 - 4129. 10.1063/1.1147558 . hal-03534502

\section{HAL Id: hal-03534502 \\ https://hal.science/hal-03534502}

Submitted on 19 Jan 2022

HAL is a multi-disciplinary open access archive for the deposit and dissemination of scientific research documents, whether they are published or not. The documents may come from teaching and research institutions in France or abroad, or from public or private research centers.
L'archive ouverte pluridisciplinaire HAL, est destinée au dépôt et à la diffusion de documents scientifiques de niveau recherche, publiés ou non, émanant des établissements d'enseignement et de recherche français ou étrangers, des laboratoires publics ou privés. 


\section{AD $\mid \begin{aligned} & \text { Review of } \\ & \text { Scientific Instruments }\end{aligned}$}

\section{Steady state behavior of a multipolar plasma device}

M. Carrère, L. Chérigier, C. ArnasCapeau, G. Bachet, and F. Doveil

Citation: Review of Scientific Instruments 67, 4124 (1996); doi: 10.1063/1.1147558

View online: http://dx.doi.org/10.1063/1.1147558

View Table of Contents: http://scitation.aip.org/content/aip/journal/rsi/67/12?ver=pdfcov

Published by the AIP Publishing

Articles you may be interested in

Steady-state direct-current plasma immersion ion implantation using a multipolar magnetic field electron cyclotron resonance plasma source

J. Vac. Sci. Technol. A 19, 2889 (2001); 10.1116/1.1412653

A dusty plasma device for producing extended, steady state, magnetized, dusty plasma columns

Rev. Sci. Instrum. 63, 5266 (1992); 10.1063/1.1143438

Collisional diffusion of a plasma in multipolar and picket fence devices

Phys. Fluids 26, 545 (1983); 10.1063/1.864139

Steady State Fracture Device

Rev. Sci. Instrum. 37, 664 (1966); 10.1063/1.1720277

SteadyState Plasma in a Plane

Phys. Fluids 8, 1139 (1965); 10.1063/1.1761366

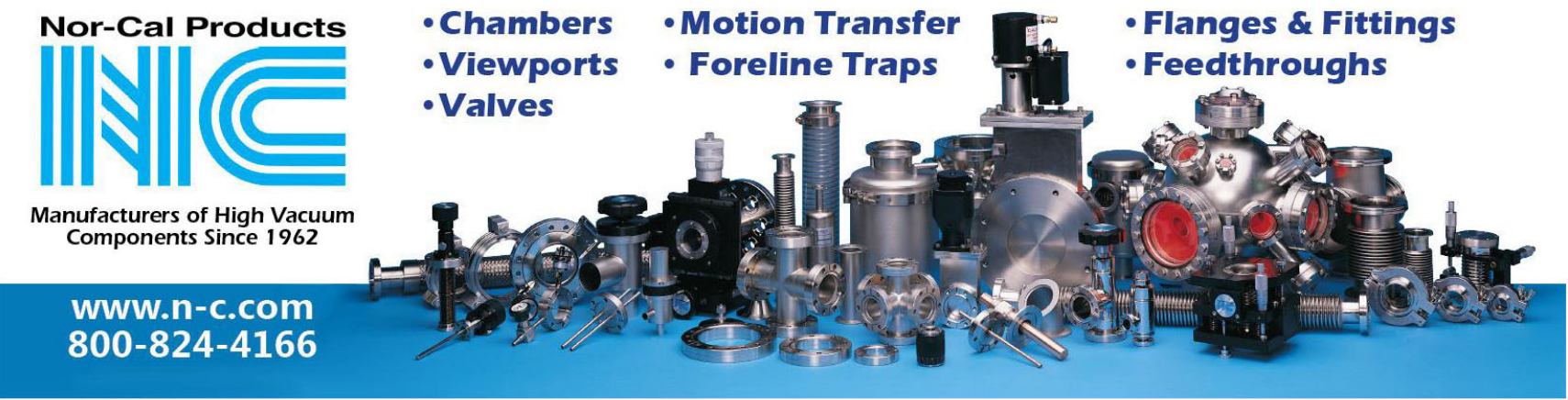




\title{
Steady state behavior of a multipolar plasma device
}

\author{
M. Carrère, L. Chérigier, C. Arnas-Capeau, G. Bachet, and F. Doveil \\ Equipe Turbulence Plasma, URA 773 C.N.R.S./Université de Provence, Institut Méditerrannéen \\ de Technologie, F-13451 Marseille Cedex 20, France
}

(Received 6 July 1995; accepted for publication 11 September 1996)

\begin{abstract}
A low pressure discharge plasma, with magnetic multipolar confinement, is produced in a cylindrical ultrahigh vacuum device. The device has been specially designed to allow both moving Langmuir probe measurements and laser induced fluorescence diagnostic for the plasma ions. It is well suited to explore the sheaths region in a bounded plasma and to study wave and turbulence in a laboratory plasma. The main characteristics of the experimental setup and of the produced plasma are described. (C) 1996 American Institute of Physics. [S0034-6748(96)01112-4]
\end{abstract}

\section{INTRODUCTION}

In experimental studies of plasma turbulence, it is often important to have a large volume unmagnetized plasma in which parameters can be varied in a controlled way. At first described by Limpaecher and MacKenzie ${ }^{1}$ two decades ago, confinement in a multipolar surface magnetic field structure yields large volumes of homogeneous, steady-state, unmagnetized plasma. In a multipolar device, the plasma is produced by an electrical discharge at low pressure in a large vacuum chamber where a series of filaments emit the primary electrons. The multipolar magnetic field at the walls repels the ionizing electrons and ensures a large enhancement of both density and uniformity of the plasma as well as a low noise level. Such plasmas have been produced for basic experiments in plasma physics ${ }^{2}$ and intense ion beam generation. ${ }^{3}$ More recently, they have been successfully used for material processing, ${ }^{4}$ hydrogenated amorphous silicon thin-film deposition, ${ }^{5}$ and plasma etching. ${ }^{6}$

In this letter we describe a multipolar plasma device designed for basic plasma turbulence study. We also would like to take the profit of the starting of this new device to try to elucidate the main phenomena that rule the creation and the steady-state behavior of the plasma. This also implies a careful exploration of the plasma sheaths in the bounded plasma. Beside the use of Langmuir probes moving in ultravacuum, the device has been specially designed to allow the use of a nonintrusive diagnostic based on laser induced fluorescence (LIF) to measure both the axial and radial ion distribution function in an extended region along the axis. This unique feature allows new careful measurements of the ion motion in the vicinity of a biased plane electrode or in the more intricate region of the multipolar magnetic field. ${ }^{7,8}$

The steady state behavior of a multipolar discharge created by hot emissive filaments is far from being understood. This article also describes a simple model based on the equation which describes the balance between creation and loss in the plasma. This model is shown to agree with experimental results and allows to determine from basic plasma parameters measurements (essentially discharge current, plasma density, and electron temperature) the plasma confinement time in various geometries, and the plasma creation rate by thermal electrons.

This article is organized as follows. In Sec. II, the experimental setup is described. Section III describes the plasma diagnostics. The characteristics of the produced plasma and some salient experimental results are given in Sec. IV.

\section{EXPERIMENTAL SETUP}

Figure 1 gives a sketch of the device. The plasma is produced in a stainless steel cylindrical vacuum vessel $45 \mathrm{~cm}$ in diameter and $80 \mathrm{~cm}$ long. The walls are cooled by water circulation. The two end sliding doors give an easy access to the plasma chamber. The vessel lies on an adjustable support to ensure its horizontality.

The main peculiarity of this vessel is the presence of three rectangular optical windows which extend along the generatrix of the cylinder. Two diametrically opposite windows allow a laser to be beamed transversally through the plasma from almost any position along the generatrix of the cylinder. The third window is allowed to collect the fluorescence outside the device at about $90^{\circ}$ to the incident beam. Figure 2 gives a transversal view of one of these windows and shows how the sealing is made between the specially designed plane Pyrex glass window and the vessel. The ion radial velocity distribution function can thus be measured at any position along the axis of the cylinder.

The incident laser beam can also be propagated longitudinally along the axis of the cylinder by means of two small optical apertures located on each of the plane end doors. By collecting the fluorescence through the same window as before, the ion axial velocity distribution function can thus, also be measured at any position along the axis of the cylin-

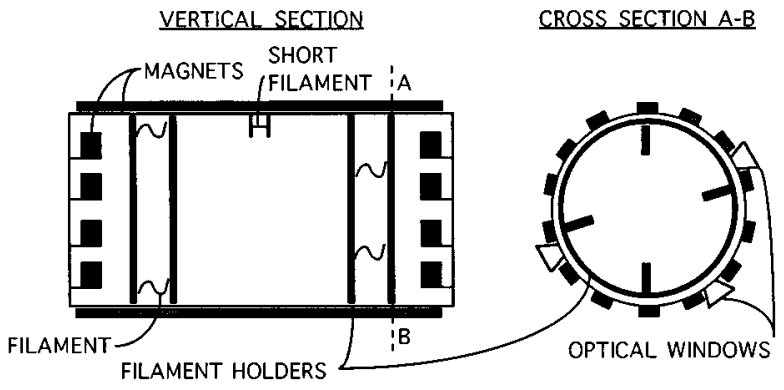

FIG. 1. Sketch of the multipolar device: filament holders and permanent magnet rows are removable. 


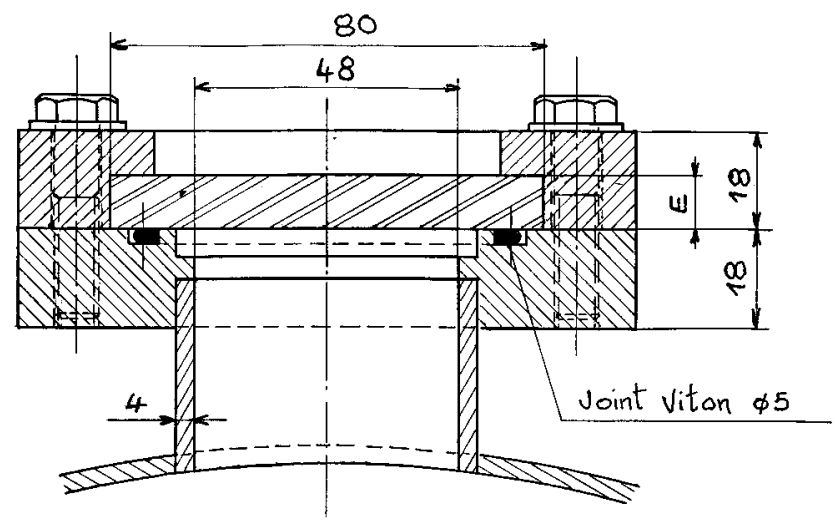

FIG. 2. Section of a rectangular window; distances are in $\mathrm{mm}$.

der. Beside these main windows, the device has a large number of flanges to allow to bring current, voltage and to install fixed or moving probes.

The cylindrical vessel is surrounded on the outside by 14 rows of permanent magnets with alternate polarizations. Each row is obtained by lining up small parallelipipedic ferrite magnets $(40 \times 65 \times 20 \mathrm{~mm}$ in size $)$ in steel sections. Inside the vessel, four lines of the same permanent magnets inserted into sealed boxes can be held in place on each of the two thick plane doors closing the ends of the cylinder. Both external and internal magnetic rows can be easily removed if one wishes. Figure 3 shows maps of the amplitude of the multipolar magnetic field measured with the probe of a Hall effect gaussmeter moving in a plane perpendicular to the axis. The amplitude is obtained from three different measurements of the magnetic field in three orthogonal directions at a given position. Close to a magnet, the magnetic field amplitude is $800 \mathrm{G}$. In Fig. 3(a) which corresponds to a plane located at $0.5 \mathrm{~cm}$ from one end door, the difference in amplitude between the alternate continuous and dashed lines is equal to $31.5 \mathrm{G}$. The influence of the magnet rows on the door is clearly observed. In Fig. 3(b) which corresponds to the simplest situation of a plane located at $26 \mathrm{~cm}$ from one end door, the difference in amplitude between the alternate continuous and dashed lines is equal to $25 \mathrm{G}$. The magnetic field free region extends on a cylinder of diameter $10 \mathrm{~cm}$ around the axis.

A turbomolecular pump is used to evacuate the device at a pressure in the range of $10^{-8}$ Torr. A Pirani gauge and an ionization gauge measure the base vacuum. This system is completely self-acting, included a nitrogen refilling process. A mass spectrometer gives the composition of the residual gasses in the vessel. Once a good base vacuum is obtained, the argon gas is introduced in the vessel through a controlled microleak at a working pressure $P$ of the order of a few $10^{-4}$ Torr.

The plasma is generated by the so-called primary electrons, which are emitted by thermionic filaments (discharge current $I_{d}$ ) and energized by the negative bias (discharge voltage $V_{d}$ ) of the filaments with respect to the grounded wall. When sufficiently energetic, these electrons undergo inelastic collisions with the neutral atoms of the gas. The result is excitation, dissociation, and ionization of the gas.
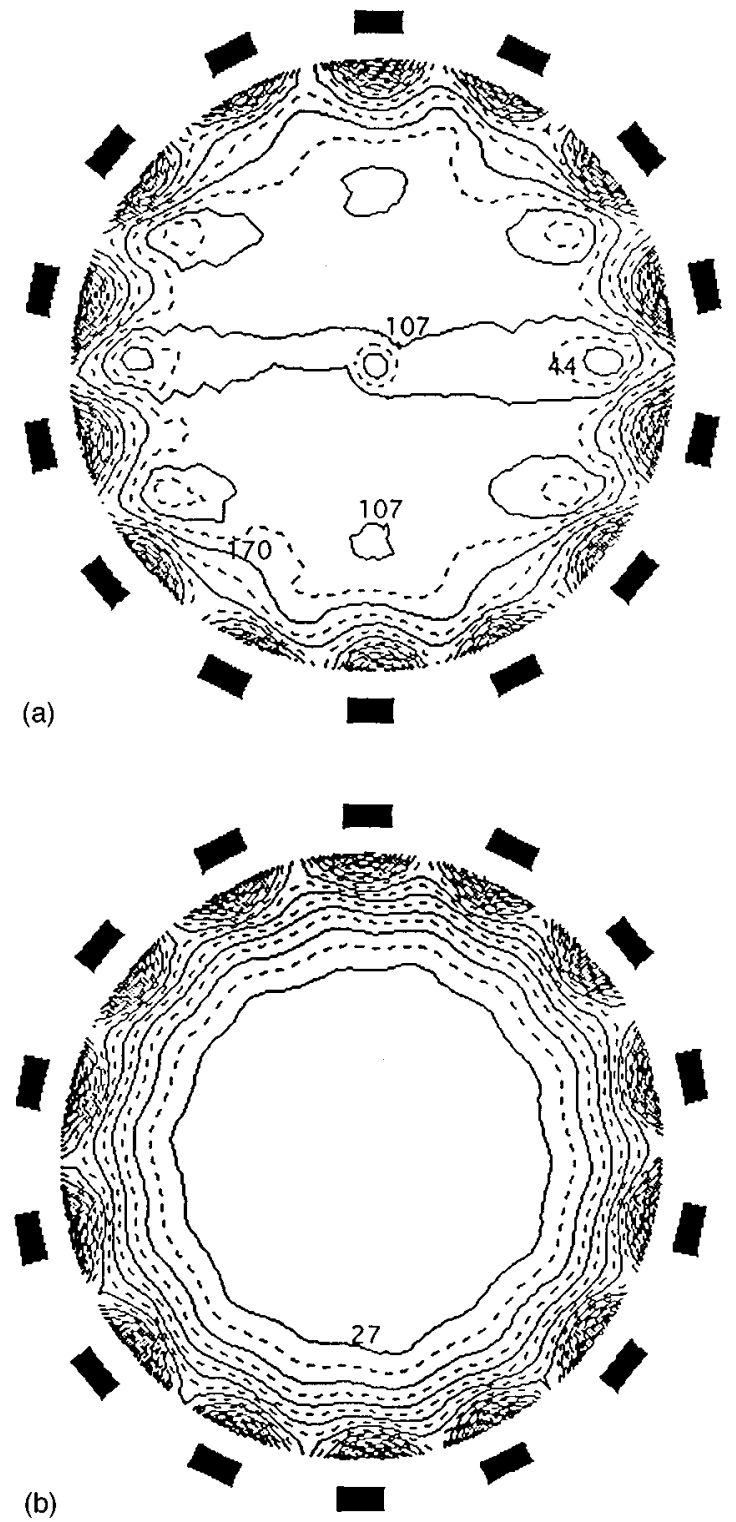

FIG. 3. Level lines for the amplitude of the measured multipolar magnetic field in a plane perpendicular to the axis (a) at $0.5 \mathrm{~cm}$ from one end door, and with $\Delta B=31.5 \mathrm{G}$; (b) at $26 \mathrm{~cm}$ from one end door, and with $\Delta B=25 \mathrm{G}$, where $\Delta B$ is the amplitude difference between a dashed and the next continuous level line.

Finally, these electrons are collected by the wall of the discharge chamber providing a return current. The two filament holders are of the same kind: they consist in two copper rings of $40 \mathrm{~cm}$ in diameter spaced $7 \mathrm{~cm}$ apart and insulated by ceramics, between which four tungsten filaments are stretched. Both filament holders are mounted on small ceramic wheels so that they can be installed vertically in any place along the axis of the device and are electrically insulated from the grounded wall. For holder No. 1, the filaments are near the inner wall inside the multipolar magnetic field. For holder No. 2, the filaments are within the plasma in a region of zero magnetic field. We will see later that all these geometric considerations are important. Typically, a plasma is obtained with a $1 \mathrm{~A}$ discharge current, $50 \mathrm{~V}$ dc discharge voltage between the grounded wall of the vessel, and one end of a filament holder. 
The device can also be operated as a double plasma (DP) machine with a source and a target plasma. A system of one to three plane grids stretched across the device axis is also mounted on small insulated ceramic wheels that allow to vary the relative length of the two plasmas. Each grid is held by two rings (about $30 \mathrm{~cm}$ in diameter) and can be independently biased. The distance between two plane grids is 1 $\mathrm{mm}$. The potential of each plasma can also be controlled by biasing an electrically insulated stainless steel plane electrode fixed on each end door. DP devices are very useful to study beam plasma instability and turbulence ${ }^{9}$ in a laboratory.

\section{PLASMA DIAGNOSTICS}

\section{A. Langmuir probes}

Several Langmuir probes are installed and measure such parameters as the electronic density and temperature, the plasma potential, and the floating potential when storing the whole probe characteristic or the plasma density fluctuations when biasing the probe at a fixed voltage. Plane and cylindrical probes of different shapes can be used. The plane probes consist of a tantalum foil disk of $\sim 5 \mathrm{~mm}$ diameter. The cylindrical probes are made of tungsten wire with a diameter of $0.1 \mathrm{~mm}$ and a typical length of $1 \mathrm{~cm}$. The wire is normally welded to the core of a coaxial connector and except for the probe tip covered with an alumina tube of small diameter to keep the disturbance of the plasma at a minimum.

To record the probe characteristic, the data acquisition system is controlled by an on-line 386 processor computer equipped with a four channel 12 bit analog digital converter (ADC), a two channel 12 bit ADC, and a digital interface port. The probe circuit mainly consists of an operational amplifier to record the probe current by monitoring the voltage drop over a probe shunt. The probe driver voltage circuit is equipped with an internal signal generator providing triangular wave form that can be internally connected to the amplifier in order to display the characteristic on the screen of an oscilloscope for monitoring purposes. In the external mode, the probe voltage is controlled by the 12-bit ADC. Two types of probe driver circuit can be used, with a maximum output voltage of $\pm 30 \mathrm{~V}$ (resp. $\pm 15 \mathrm{~V}$ ) and a maximum output current of $20 \mathrm{~mA}$ (resp. $2 \mathrm{~A}$ ). The input voltage range of the $\mathrm{ADC}$ is $\pm 10 \mathrm{~V}$ with 11 bit linearity. The gain of the amplifier can be set digitally from 1 to 10 and 100. In order to utilize the full resolution of the ADC an additional analogic amplifier with a gain varying from 1 to 10 by step of 1 can be inserted in the circuit. Although the cutoff frequency of the amplifier is high enough to record low-frequency fluctuations, the cutoff frequency of the present design is reduced to $1 \mathrm{kHz}$ to improve the stability of the circuit. The probe characteristic can be scanned with a high resolution of about 500 or 1000 points. In order to perform digital derivation of the characteristic, the possibility of averaging over each point has been implemented.

One of the probes is fixed at the tip of an UHV translation and rotation manipulator. It can thus be moved longitudinally and can also be rotated to explore a slice of plasma.
One other probe can analyze the plasma along a radius. The positioning of the probes is controlled by the PClab card and commercial stepping motors.

\section{B. Laser induced fluorescence diagnostic}

Laser induced fluorescence ${ }^{10}$ is a nonintrusive technique, based on the Doppler effect, ${ }^{11}$ which allows a very accurate measurement of the velocity profile of the ion distribution function in a plasma. By scanning the $\mathrm{cw}$ beam of a single frequency dye laser across the line corresponding to the transition $3 d^{2} G_{9 / 2} \rightarrow 4 p^{2} F_{7 / 2}$ of $\mathrm{Ar}^{\mathrm{II}}$ at $611.492 \mathrm{~nm}$, every velocity class of ions in the metastable level $\left(3 d^{2} G_{9 / 2}\right)$ can be explored. The upper level decays toward the lower one with a fluorescent emission at $460.957 \mathrm{~nm}$. The scanning laser pulsation and wave number define the locality in ion velocity space $\bar{v}$. Locality in space $\bar{r}$ is obtained by collecting the fluorescence at $90^{\circ}$ to the incident laser beam. Thus the diagnostic allows to accurately probe the total ion phase space $(\bar{r}, \bar{v})$.

The laser induced fluorescence diagnostic uses a cw 899-21 tunable ring dye laser from Coherent pumped by an Inova 306 argon ion laser. By moving a plane mirror attached to a slide, the laser beam can be very easily propagated longitudinally or transversally through the plasma. The laser beam is chopped and a lock-in amplifier is used to discriminate the fluorescent signal from the spontaneous emission of the plasma at the same frequency. A narrow band pass interferential optical filter $(1 \mathrm{~nm})$ is used to eliminate unwanted radiation. An $f / 2$ lens collects the fluorescence and forms, with a magnification of 0.6 , the laser beam image on a $1 \mathrm{~mm}$ diam diaphragm, which sets the spatial resolution of the diagnostic at $6 \mathrm{~mm}^{3}$. A small part of the laser output is sent through an iodine cell and the iodine absorption gives an absolute calibration of the laser emission.

The entire diagnostic is under the control of an on-line 386 microprocessor computer. The laser scan is monitored and at each step of the scanning process the data on the level of iodine absorption on one hand and that of the fluorescence on the other hand are stored to 12 bits of accuracy. This computer is also used for processing the data.

Although the laser linewidth is $0.5 \mathrm{MHz}$, which corresponds to a resolution in velocity of $0.3 \mathrm{~ms}^{-1}$, we have restricted, for convenience, the frequency acquisition sampling to reach a "practical" resolution of $30 \mathrm{~ms}^{-1}$.

\section{EXPERIMENTAL RESULTS}

As compared to electrostatic confinement, multipolar confinement is allowed to create a homogeneous and quiescent plasma for a low energy cost. Besides the geometric configuration of the device, three control parameters rule the steady-state behavior of the plasma. These are the gas pressure $P$, the discharge voltage $V_{d}$, and the temperature of the filaments, through the heating current $I_{f}$. With Langmuir probes we have explored the main plasma parameters (density, electron temperature) in standard working conditions $\left(10^{-5}<P<10^{-3}\right.$ Torr, $\left.0<I_{d}<2 \mathrm{~A}\right)$.

As was previously established, ${ }^{12}$ multipolar confinement mainly acts on the primary electrons. Due to multiple reflec- 
tion in the magnetic mirrors, the primary electrons travel a distance $L$ larger than the plasma typical size before being lost. For the low pressure regime which we consider, the plasma is essentially collisionless and the mean free path for an ionizing collision $\lambda$ is also larger than the typical size of the plasma. Assuming that the plasma is mainly created through ionizing collisions of the primary electrons, and equating plasma creation to plasma loss, one can therefore write an equilibrium equation ${ }^{13}$ for the plasma density $n$ :

$$
\rho I_{d} / e \wp=n / \tau,
$$

where: $\rho$ is the probability for a primary electron to perform an ionizing collision, $I_{d}$ is the primary electron current, $e$ is the elementary charge, $\wp$ is the plasma volume, and $\tau$ is the plasma confinement time.

In the above equation, the primary electron current is taken to be equal to the discharge current $I_{d}$ since, due to the small filament area, the ion current accelerated toward it is negligible with respect to the thermoelectron current. The ionizing collision mean free path $\lambda$ is given by

$$
\lambda=\left[n_{0} \sigma\left(V_{d}\right)\right]^{-1},
$$

where $n_{0}$ is the neutral gas density and $\sigma\left(V_{d}\right)$ is the ionizing collision cross section. As long as $\lambda$ is larger than the plasma size ( $\lambda$ is equal to about $11 \mathrm{~m}$ for $P=10^{-4}$ Torr and $V_{d}=50$ $\mathrm{V})$, the effective primary electron distance $L$ is independent of the neutral gas density $n_{0}$ and

$$
\rho=1-\exp (-L / \lambda) \text {. }
$$

Combining Eqs. (1), (2), and (3), we get

$$
n / I_{d}=(\tau / e \wp)\left\{1-\exp \left[-n_{0} \sigma\left(V_{d}\right) L\right]\right\} .
$$

Previous measurements ${ }^{1,12}$ indicate that the confinement time $\tau$ essentially depends on the plasma size. It can therefore be considered as a constant in Eq. (4).

The above hypotheses are confirmed by Fig. 4(a), where the ratio $n / I_{d}$ obtained experimentally is plotted versus the argon gas pressure $P$ (equivalent to $n_{0}$ ). The plasma density is measured at a given point in the center of the discharge. Four long spring shaped filaments are used on each filament holder and $V_{d}=50 \mathrm{~V}$. Black dots (resp. white dots) correspond to the case where filament holder No. 1 (resp. No. 2) is used to create the plasma, with the filaments lying inside (resp. outside) the multipolar magnetic field region. In both cases, we observe the same behavior predicted by Eq. (4): the plasma density increases linearly with the discharge current and the ratio of these two quantities saturates when the gas pressure increases. The continuous curves of Fig. 4(a) are obtained by fitting the experimental results with a curve given by Eq. (4). Since the equation fits the data quite well, we can first conclude that $n$ must be proportional to $I_{d}$. For the case where the filaments are in the plasma, and outside the magnetic field, the best fit is obtained for $\tau=267 \mu \mathrm{s}$ and $L=2.8 \mathrm{~m}$. For the case where the filaments lie inside the magnetic field region, $\tau=245 \mu \mathrm{s}$ and $L=1.5 \mathrm{~m}$. Both cases give approximately the same value for the confinement time whereas the effective length for the primary electrons, equal to about 7 times the device diameter when the filaments are inside the plasma, is smaller when their emission is per-
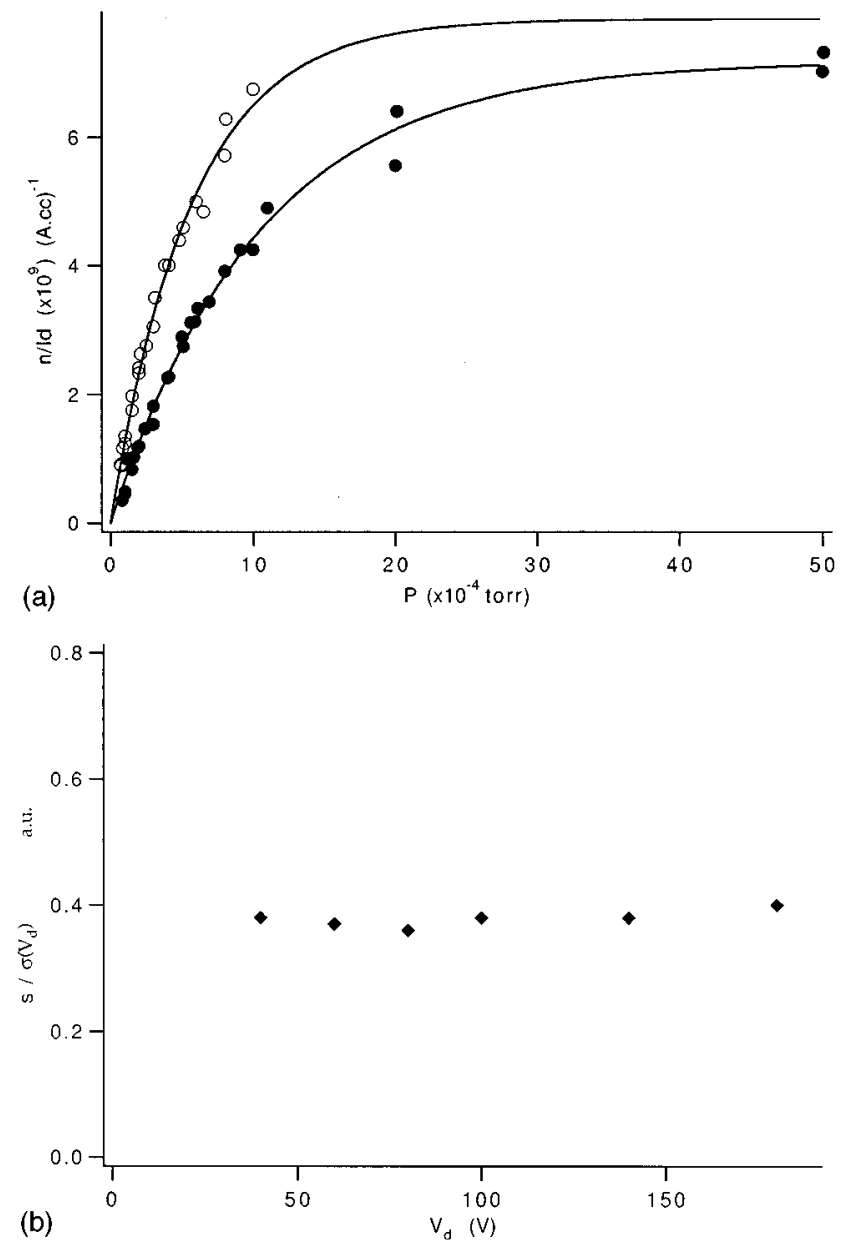

FIG. 4. (a) Density variation (normalized to discharge current) as a function of the argon gas pressure. Black (resp. white) dots correspond to filament holder No. 1 (resp. 2). $V_{d}=50 \mathrm{~V}$. (b) Initial slope of $n / I_{d}$ vs $P$ [normalized to $\left.\sigma\left(V_{d}\right)\right]$ for various values of $V_{d}$. Filament holder No. 1 .

turbed by the multipolar magnetic field. A similar analysis performed with filament holder No. 2 after removing the permanent magnets from one of the end doors shows that $\tau$ is unchanged and $L$ becomes the same order as the plasma size, so that the influence of the magnetic confinement on the primary electrons is considerably reduced. In the low pressure limit, Eq. (4) predicts that the ratio $n / I_{d}$ increases linearly with pressure with a slope $s$ proportional to $\tau L \sigma\left(V_{d}\right)$. Figure 4(b) gives $s / \sigma\left(V_{d}\right)$ when $V_{d}$ varies, for filament holder No. 1. If we again assume that $\tau$ is a constant, this result shows that $L$ is essentially independent of $V_{d}$.

The electron plasma temperature $T_{e}$ is obtained by analyzing the characteristics of a probe located at the center of the device. Such an analysis often reveals the presence of two electron populations with different temperatures: a bulk plasma population and a higher energy tail. By $T_{e}$ we refer to the bulk plasma electrons with the lowest temperature. Figure 5 shows the variation of $T_{e}$ as a function of the gas pressure. The black (resp white) dots correspond to the case where filament holder No. 1 (resp No. 2) is used. Two different values of the filament temperature are considered as indicated by square and circle dots. Figure 5 does not show any significant dependency of the plasma temperature on the 


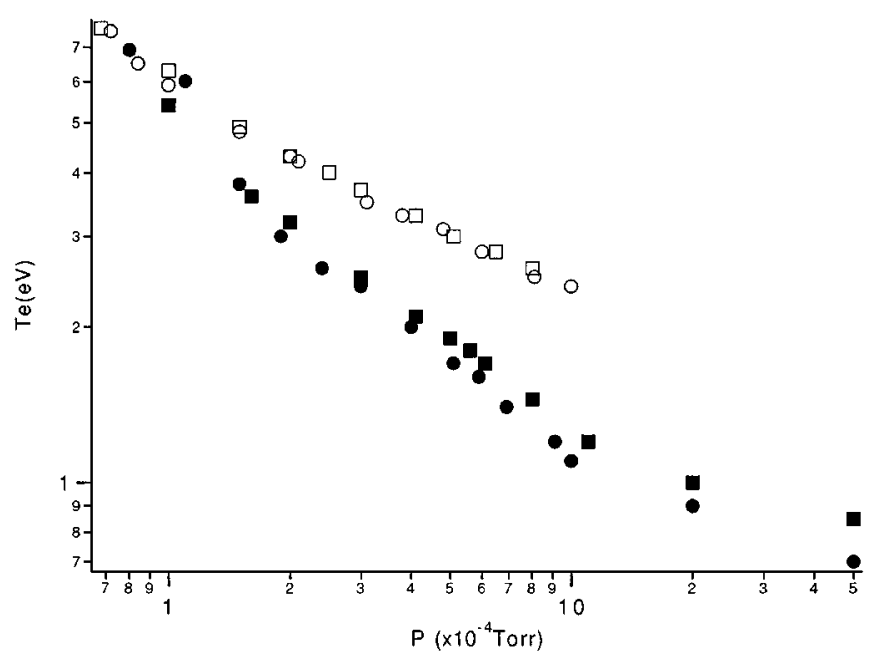

FIG. 5. Electron temperature variation vs argon gas pressure. Black (resp. white) dots correspond to filament holder No. 1 (resp. 2). $V_{d}=50 \mathrm{~V}$. Squares: current filament $I_{f}=27 \mathrm{~A}$; circles: $I_{f}=26 \mathrm{~A}$.

filament temperature since the difference between square and circular points is well within experimental uncertainty. In the case where the filaments lie outside the multipolar magnetic field region (filament holder No. 2) the temperature is higher than when the filaments are inside the magnetic field region. In both cases, the temperature decreases with pressure according to a negative power law. Such a behavior can be easily understood. The rate of plasma creation by thermal electrons is characterized by a time

$$
\tau_{\mathrm{th}}=\left(n_{0}\langle\sigma v\rangle_{\mathrm{th}}\right)^{-1},
$$

where $n_{0}$ is the neutral gas density and $\langle\sigma v\rangle_{\text {th }}$ is the coefficient for ionizing collisions by thermal electrons which depends on the electron energy distribution function. Using Bohm's criterion, ${ }^{14}$ the rate of plasma loss can be classically related to the plasma temperature

$$
\tau=\wp / S c_{S},
$$

where $S$ is the total surface of the anode and $c_{s}=\left(\kappa T_{e} / M\right)^{1 / 2}$ is the ion acoustic velocity ( $M$ is the ion mass and $\kappa$ the Boltzmann constant). The balance equation that describes the equilibrium of the plasma simply states that the loss plasma rate $1 / \tau$ is equal to the creation rate by the thermal plasma electrons $1 / \tau_{\text {th }}$ increased by the creation rate by the primary electrons. The equilibrium plasma temperature is obtained when the rate of plasma production by primary electrons, therefore equal to $1 / \tau-1 / \tau_{\text {th }}$, is maximum. Assuming a power law for the variation of $\langle\sigma v\rangle_{\text {th }}$ with temperature (expressed in $\mathrm{eV}$ ), and using the experimental power law given by Fig. 5 , we obtain $\langle\sigma v\rangle_{\mathrm{th}}=3 \times 10^{-12} T_{e}^{2.8} \mathrm{cc} / \mathrm{s}$ for the white dots and $\langle\sigma v\rangle_{\mathrm{th}}=24.1 \times 10^{-12} T_{e}{ }^{1.9} \mathrm{cc} / \mathrm{s}$ for the black dots. If the plasma electrons were maxwellian, we would have gotten $\langle\sigma v\rangle_{\mathrm{th}}=80 \times 10^{-12} T_{e}^{2.4} \mathrm{cc} / \mathrm{s}$ in the considered energy range. ${ }^{15}$ To control externally the electron temperature at a given gas pressure, a Maxwell demon, ${ }^{16}$ made of a tangle of a fine conducting wire, can also be used.

The ion velocity distribution function is measured with the LIF diagnostics. Figure 6 shows the axial velocity distribution measured at various distances of a plane metallic plate

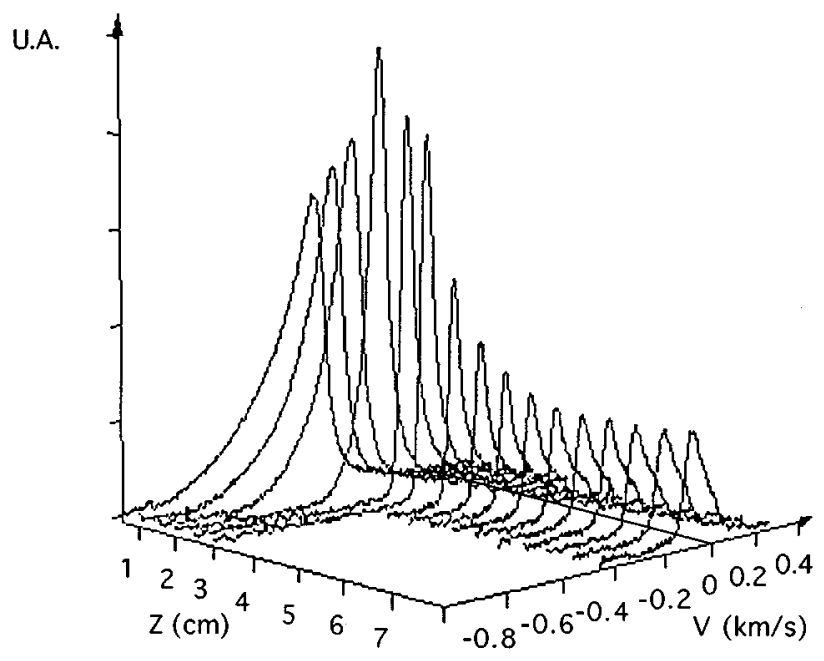

FIG. 6. Measured ion axial velocity distribution function as a function of the distance from a floating metallic plate.

at floating potential $V_{f}=-40 \mathrm{~V}$. The laser beam enters the plasma through a small hole of diameter $4 \mathrm{~cm}$ in the plate. Far from the plate, the ion distribution function can be accurately described by a centered maxwellian of temperature $T_{i}=0.04 \mathrm{eV}$, to be compared to the $0.025 \mathrm{eV}$ temperature of the neutral argon gas at room temperature. The same result applies for the measured radial velocity distribution function. When getting closer to the plate, the influence of the electrostatic boundary potential of the plasma is observed, until inside the plasma sheath: the main effect is a drift associated to the acceleration of the ions in the decreasing potential and a careful analysis of the curves shows a good qualitative agreement $^{8}$ with a simple analytical model. However, a strong heating of the ions is also observed. It may be related to the presence of sheath oscillations and remains to be explained. The LIF technique appears as a powerful non intrusive diagnostic for ions in a plasma discharge with density as low as $10^{8} \mathrm{cc}^{-1}$.

A large volume homogeneous unmagnetized discharge plasma can thus be produced in the central region of this multipolar plasma device. Beside its great flexibility and the use of ultrahigh vacuum technology to eliminate impurities which can perturb wave propagation in a low pressure discharge, the device has been specially designed to allow to apply a nonintrusive laser induced fluorescence diagnostic to measure the local ion velocity distribution function with an unprecedented resolution. ${ }^{17}$ The role of the control parameters (gas pressure, discharge voltage, and temperature of the filaments) and the influence of the position of the filaments on the steady state behavior of the plasma has been investigated. If a global understanding of the discharge has been obtained, there still remains a lot of basic questions that need to be solved to attain a detailed description of the plasma: hysteresis observed on the current-voltage characteristics of the discharge, ${ }^{18}$ plasma-sheaths regions and their role on the apparition of self-oscillations, ${ }^{19}$ and transition to chaos ${ }^{20}$ in the discharge, thermalization of electrons in the absence of collisions. ${ }^{18}$ Beside experimental study of wave propagation and turbulence, the present device appears as a useful tool in 
the experimental investigation of these basic problems of bounded plasmas.

\section{ACKNOWLEDGMENTS}

The technical assistance of R. Bompard, J. C. Chezeaux, L. Roussel, B. Squizzaro, and A. Totin is greatly appreciated. During this work, L.C. and M.C. benefited from grants of the Ministère de la Recherche et de la Technologie.

${ }^{1}$ R. Limpaecher and K. R. MacKenzie, Rev. Sci. Instrum. 44, 726 (1973).

${ }^{2}$ F. Doveil, Y. Vosluisant, and S. I. Tsunoda, Phys. Rev. Lett. 69, 2074 (1992).

${ }^{3}$ W. L. Stirling, P. M. Ryan, C. C. Tsai, and K. N. Leung, Rev. Sci. Instrum. 50, 102 (1979).

${ }^{4}$ S. Gourrier, A. Mircea, and M. Bacal, Thin Solid Films 65, 315 (1980).

${ }^{5}$ B. Drevillon, J. Huc, A. Lloret, J. Perrin, G. de Rosny, and J. P. M. Schmitt, Appl. Phys. Lett. 37, 646 (1980).

${ }^{6}$ T. D. Mantei and T. Wicker, Appl. Phys. Lett. 43, 84 (1983).

${ }^{7}$ G. Bachet, L. Chérigier, M. Carrère, and F. Doveil, Phys. Fluids B 5, 3097 (1993).
${ }^{8}$ G. Bachet, L. Chérigier, and F. Doveil, Phys. Plasmas 2, 1782 (1995).

${ }^{9}$ F. Doveil and D. Grésillon, Phys. Fluids 18, 1756 (1975).

${ }^{10}$ R. Measures, J. Appl. Phys. 39, 5232 (1968); R. A. Stern and J. A. Johnson, Phys. Rev. Lett. 34, 1548 (1975).

${ }^{11}$ D. N. Hill, S. Fornaca, and M. G. Wickham, Rev. Sci. Instrum. 54, 309 (1983).

${ }^{12}$ J. M. Buzzi, J. Snow, and J. L. Hirsfield, Phys. Lett. 54A, 344 (1975).

${ }^{13}$ A. Truc, T. D. Mantei, and D. Grésillon, Appl. Phys. Rev. 12, 1143 (1977).

${ }^{14}$ D. Bohm, in The Characteristics of Electrical Discharges in Magnetic Fields, edited by A. Guthrie and R. K. Wakerling (McGraw-Hill, New York, 1949).

${ }^{15}$ A. R. Martin, Physica 58, 77 (1972).

${ }^{16}$ K. R. MacKenzie, R. J. Taylor, D. B. Cohn, E. R. Ault, and H. Ikezi, Appl. Phys. Lett. 18, 529 (1971).

${ }^{17}$ L. Chérigier, Thèse de l'Université de Provence, Marseille, 1994.

${ }^{18}$ M. Carrère, Thèse de l'Université de Provence, Marseille, 1994.

${ }^{19}$ C. Arnas-Capeau, G. Bachet, and F. Doveil, Phys. Plasmas 2, 4650 (1996).

${ }^{20}$ C. Arnas-Capeau, G. Prasad, G. Bachet, and F. Doveil, Phys. Plasmas 3, 3331 (1996). 